\title{
Optical pulsations from a transitional millisecond pulsar
}

F. Ambrosino ${ }^{1, *}$, A. Papitto ${ }^{2, * \dagger}$, L. Stella ${ }^{2}$, F. Meddi ${ }^{1}$, P. Cretaro ${ }^{3}$, L. Burderi ${ }^{4}$, T. Di Salvo ${ }^{5}$, G. L. Israel ${ }^{2}$, A. Ghedina ${ }^{6}$, L. Di Fabrizio ${ }^{6}$ \& L. Riverol $^{6}$

${ }^{1}$ Dipartimento di Fisica, Università di Roma “La Sapienza”, Piazzale Aldo Moro, 5, 00185 Roma, Italy.

${ }^{2}$ INAF - Osservatorio Astronomico di Roma, Via Frascati, 33, 00040 Monte Porzio Catone (RM), Italy.

${ }^{3}$ INFN - Sezione di Roma 1, Piazzale Aldo Moro, 5, 00185 Roma, Italy.

${ }^{4}$ Dipartimento di Fisica, Università di Cagliari, SP Monserrato-Sestu, Km 0.7, I-09042 Monserrato, Italy

${ }^{5}$ Dipartimento di Fisica e Chimica, Università di Palermo, via Archirafi 36, 90124 Palermo, Italy

${ }^{6}$ Fundación Galileo Galilei - INAF, Rambla José Ana Fernández Pérez, 7, 38712 Breña Baja, TF, Spain.

*These authors contributed equally to this work.

†corresponding author (e-mail: alessandro.papitto@oa-roma.inaf.it)

Millisecond pulsars are neutron stars that attain their very fast rotation during a $10^{8}-10^{9} \mathbf{~ y r}-$ long phase of disk-accretion of matter from a low mass companion star ${ }^{\sqrt[1]{12}}$. They can be detected as accretion-powered millisecond X-ray pulsars if towards the end of this phase their magnetic field is strong enough to channel the in-flowing matter towards their magnetic poles $^{3}$. When mass transfer is reduced or ceases altogether, pulsed emission generated by 
magnetospheric particle acceleration and powered by the star rotation is observed, preferentially in the radio ${ }^{4}$ and gamma-ray ${ }^{\sqrt{5}}$ bands. A few transitional millisecond pulsars that swing between an accretion-powered X-ray pulsar regime and a rotationally-powered radio pulsar regime in response to variations of the mass in-flow rate have been recently identified 6 . Here we report the detection of optical pulsations from a transitional pulsar, the first ever from a millisecond spinning neutron star. The pulsations were observed when the pulsar was surrounded by an accretion disk, and originated inside the magnetosphere or within a few hundreds of kilometers from it. Energy arguments rule out reprocessing of accretionpowered X-ray emission and argue against a process related to accretion onto the pulsar polar caps; synchrotron emission of electrons in a rotation-powered pulsar magnetosphere ${ }^{8}$ seems more likely.

PSR J1023+0038 is a $1.69 \mathrm{~ms}$ spinning neutron star in a $4.75 \mathrm{hr}$ orbit around a $0.2 \mathrm{M}_{\odot}$ main-sequence-like companion, located at a distance ${ }^{9}$ of $1.37 \mathrm{kpc}$. It was discovered in 2008 as a rotationally-powered radio pulsar ${ }^{\sqrt{6}}$ releasing a spin down power ${ }^{\sqrt{9}}$ of $4.3 \times 10^{34} \mathrm{erg} \mathrm{s}^{-1}$, corresponding to a surface magnetic field of $\simeq 10^{8} \mathrm{G}$. In June 2013 radio pulsations disappeared and an accretion disk developed, accompanied by a factor of $\sim 10$ increase both in the average ${ }^{\sqrt{10}+12}$ and pulsed X-ray flux ${ }^{13} \mid 60$ as compared to the radio pulsar regime. This increase was ascribed to channeled mass accretion onto the pulsar polar caps, even though the X-ray luminosity ${ }^{15}\left(\simeq 7 \times 10^{33} \operatorname{erg~s}^{-1}\right.$, 0.3-79 keV) was considerably lower than both the luminosity of accreting millisecond pulsars in outburst, and the luminosity corresponding to the mass accretion rate required to overcome the centrifugal barrier of the pulsar rotating magnetosphere and its propelling effect (assuming matter 
reaches the neutron star). Its gamma-ray ${ }^{10}$ and flat-spectrum continuum radio emission, consistent with a jet-like outflow $\frac{16}{16}$ add to the complex phenomenology of PSR J1023+0038 in the accretion disk state, possibly being manifestations of the interaction between the disk, the pulsar magnetosphere and its wind $\frac{10,15,17}{18}$. Archival observations ${ }^{6}$ indicate that PSR J1023+0038 was in a similar state in 2001. The optical counterpart of PSR J1023+0038, AY Sex, in the disk state is a $g \simeq 16.7$ mag blue object that emits an average luminosity $18\left[19\right.$ of $L_{\text {opt }} \simeq 10^{33} \mathrm{erg} \mathrm{s}^{-1}$ in the 320-900 nm band. Most of the optical/UV emission originates from the outer regions of the disk, and from the companion star's face illuminated by the pulsar high-energy radiation, which drives a $\Delta g \simeq 0.4$ mag modulation $\frac{18}{18}$ of the optical flux at the orbital period of the system.

During a 4 hour-long observation carried out on 2016, March 2-3 with SiFAP, a fast photometer with $25 \mathrm{~ns}$ time resolution that was mounted at the $3.58 \mathrm{~m}$ INAF's Telescopio Nazionale Galileo (TNG) in La Palma, Spain (see Methods), we discovered optical (320-900 nm) pulsations at the spin period of PSR J1023+0038 (see Fig. 1). In order to detect the signal we corrected the arrival times of the optical photons for the light travel time delays introduced by the pulsar orbit by using the ephemeris derived from the X-ray pulsations ${ }^{20}$ (see Tab. 1). PSR J1023+0038 was in a disk dominated state at the time of the SiFAP measurement, as inferred from Swift Xray Telescope (XRT) observations performed within a few days from the optical observations (see Methods). The optical pulse profile comprises two peaks with a fractional amplitude that varied significantly over 20 minutes-long time intervals between a maximum value of $A=(0.80 \pm 0.07) \%$ of the total average emission, and values below the detectability level ( $A<0.19 \%$; see Fig. 2 ). The maximum observed pulsed flux corresponds to $L_{\text {pulsed }} \approx 0.01 L_{\text {opt }} \approx 10^{31} \mathrm{erg} \mathrm{s}^{-1}$. The lack 
of simultaneous X-ray observations prevented us from searching for possible changes taking place at the time-scales set by the variability of the X-ray pulse amplitude ${ }^{13}$.

The region responsible for the optical pulsations cannot be larger than $c P_{\text {spin }} \sim 500 \mathrm{~km}$, as light propagation delay would smear them out. Comparison of the projected semi-major axis $a \sin i$ and epoch of the pulsar passage at the ascending node $T_{a s c}$ derived from the orbital modulation of the optical pulse period with the ephemeris derived from X-ray pulsations, showed that the region emitting the optical pulsation must be centered within $\delta r_{\rho} \sim \sigma_{a} \simeq 30 / \sin (i) \mathrm{km}$ and $\delta r_{\theta} \sim 2 \pi a \sigma_{T_{a s c}} / P_{\text {orb }} \simeq 300 / \sin (i) \mathrm{km}$ of radial and azimuthal distance from the X-ray pulsar, respectively (see Table 1 and Methods). These values are comparable to or somewhat larger than than both the $r_{c o r} \sim 24 \mathrm{~km}$ corotation radius (for a $1.4 \mathrm{M}_{\odot}$ neutron star) of PSR J1023+0038, i.e the radius where the angular velocity of the pulsar magnetosphere equals the Keplerian velocity, and the $r_{l c} \sim 80 \mathrm{~km}$ light cylinder radius where closed magnetic field lines travel at the speed of light.

Reprocessing of the X-ray pulsations at the surface of the companion star and/or in the outer disk region, as observed in some X-ray binaries hosting a strongly magnetized and slowly rotating accreting pulsar ${ }^{21}$, is ruled out as the origin of the optical pulsations from PSR J1023+0038; the reprocessing regions would have a very different light travel time-delay orbital signature than that of a region $\delta r \simeq 30-300 / \sin (i) \mathrm{km}$ away from the neutron star, and their size would greatly exceed the maximum beyond which pulsations are washed out $\left(c P_{\text {spin }} \sim 500 \mathrm{~km}\right)$. The above problems could be circumvented if the X-ray pulsations were reprocessed in the innermost regions 
of the disk close to the boundary with the pulsar magnetosphere at $r_{c o r}$. However, reprocessing of the X-ray luminosity of PSR J1023+0038 $\left(L_{X}=7 \times 10^{33} \mathrm{erg} \mathrm{s}^{-1}\right)$ by an area of $\sim \pi r_{\text {cor }}^{2}$ located at a distance $r_{\text {cor }}$ from the pulsar, would convert to a brightness temperature of $T_{i r r}=$ $\left(L_{X} / 4 \pi \sigma r_{c o r}^{2}\right) \simeq 1.1 \times 10^{6} \mathrm{~K}$. The optical output would be more than five thousands times smaller than the observed pulsed flux, ruling out also this interpretation.

If X-ray pulsations observed in the disk state are due to channeled accretion onto the magnetic polar regions of the neutron stal ${ }^{13,22}$, one may wonder whether the same polar hotspots could give rise to the the observed optical pulses. The X-ray spectrum of accreting millisecond pulsars is modeled with unsaturated Comptonisation of soft $0.5-1 \mathrm{keV}$ photons emitted from the polar hotspots, by thermal electrons with temperature of tens of $\mathrm{keV}$ presumably located in the accretion column 23 . Cyclotron emission by the same electrons in the $B_{s} \simeq 10^{8} \mathrm{G}$ magnetic field of this pulsar takes place at $E_{c y c} \simeq 1\left(B_{s} / 10^{8} \mathrm{G}\right) \mathrm{eV}$ energies. Optical pulsed flux might result from self-absorbed cyclotron emission in the optically-thick regime, with a Rayleigh-Jeans spectrum at the temperature of the Comptonising electrons extending over a range of cyclotron harmonics 24 . We adopt for PSR J1023+0038 an electron temperature $k T_{e l}$ of $100 \mathrm{keV}$ (photons up to $\sim 80 \mathrm{keV}$ have been detected from $\mathrm{i}^{[12}$ ) and an accreting polar region of conservatively large area $A \simeq \pi R_{*}^{2}\left(R_{*} / R_{\text {cor }}\right) \simeq 100 \mathrm{~km}^{2}$ (ref. ${ }^{24(25)}$ ), where $R_{*}=10 \mathrm{~km}$ is the neutron star radius. The maximum pulsed luminosity in the visible band is

$$
L_{c y c} \simeq 3 \times 10^{29}\left(\frac{A}{100 \mathrm{~km}^{2}}\right)\left(\frac{k T_{e l}}{100 \mathrm{keV}}\right) \mathrm{erg} \mathrm{s}^{-1},
$$

more than $\sim 30$ times lower than the observed pulsed luminosity, $L_{\text {pulsed }} \approx 10^{31} \mathrm{erg} \mathrm{s}^{-1}$. This does not favour an interpretation of the optical pulses in terms of cyclotron emission from electrons in 
the accretion columns; a detailed modelling will assess whether a larger optical efficiency can be achieved.

Finally we explore the rotation-powered regime of pulsars. Synchrotron radiation from secondary relativistic electrons and positrons in the magnetosphere of pulsars is generally believed to produce non-thermal pulsed emission from the optical to the X-ray band 2627 . Pulsed emission in the visible band has been detected from five rotation-powered pulsars ${ }^{828}$. They are all isolated, high-magnetic field $\left(>10^{12} \mathrm{G}\right)$ pulsars with young to moderate ages of $10^{3}-10^{5} \mathrm{yr}$. Their efficiency in converting spin down power to optical pulsed luminosity spans a broad range from $\eta_{\text {opt }} \sim 5 \times 10^{-6}$ (e.g., the Crab pulsar) to $\sim 2 \times 10^{-9}$ (see red circles in Fig. 3 and Supplementary Table ). The efficiency of PSR J1023+0038, $\eta_{\text {opt }} \sim 2 \times 10^{-5}$, is higher; note that two middleaged pulsars with a spin down power comparable to that of PSR J1023+0038 (e.g. Geminga) have $\eta_{\text {opt }} \lesssim 10^{-7}$. A few more pulsar optical counterparts were proposed based on the positional coincidence of an optical source (blue circles in Fig. 3, see Supplementary Table 1); an increasingly higher efficiency is observed for system with lower and lower spin down power and ages exceeding $10^{6} \mathrm{yr}$.

Comparison of PSR J1023+0038 - the first optical millisecond pulsar ever detected - with other rotation-powered millisecond pulsars is necessarily limited. A recent search $\frac{50}{}$ did not detect optical pulses down to a magnitude of $g \sim 25$ from PSR J0337+1715, a millisecond radio pulsar of similar spin down power and distance as PSR J1023+0038, whose average optical pulses correspond to $g \simeq 22.5$. A candidate optical counterpart of PSR J2124-3358, a close-by $4.4 \mathrm{~ms}$ 
binary radio pulsar with spin-down power $\sim 6$ times lower than that of PSR J1023+0038, has been recently reported 5 ; ; its optical luminosity of $\sim 10^{27} \mathrm{erg} \mathrm{s}^{-1}$ gives $\eta_{\text {opt }} \sim 10^{-7}$. Therefore the optical efficiency of PSR J1023+0038 appears to be orders of magnitude higher than that of other millisecond pulsars, and lower only than the efficiency of the proposed optical counterpart ${ }^{54}$ of the old $\left(1.7 \times 10^{8} \mathrm{yr}\right)$, isolated $0.8 \mathrm{~s}$ pulsar, PSR J0108-1431.

The feature that singles out PSR J1023+0038 among rotation-powered pulsars is the presence of an accretion disk. If the disk does not prevent rotation-powered pulsar mechanisms from working, the interaction between pulsar magnetosphere ${ }^{15 / 32}$ and/or its wind ${ }^{10}[17 \mid 18$ with the disk plasma may evaporate enough material to disperse radio pulsations and make them unobserved. Moreover, it would form a shock where electrons and positrons could be accelerated to the energies $(1-20 \mathrm{MeV})$ required to radiate optical synchrotron photons, in the field of $B \approx 2 \times 10^{5}-10^{6} \mathrm{G}$ of the magnetospheric region inward of the light cylinder of PSR J1023+0038. This might be the reason for the higher $\eta_{\text {opt }}$ of PSR J1023+0038. The absence of a dramatic change of the spin-down rate when the source transitioned from a radio pulsar to a disk-dominated state ${ }^{20}$ might provide additional evidence that a rotation-powered mechanism is working in PSR J1023+0038, despite the presence of a disk.

Our discovery of optical pulsations from PSR J1023+0038 demonstrates that the magnetosphere of old, weakly magnetic and quickly spinning neutron stars can give rise to such signals when surrounded by an accretion disk. This new observational window provides a promising diagnostic to probe the physics of millisecond pulsars in close binary systems, and discover new 
millisecond pulsars in low-mass X-ray binaries and in unidentified gamma-ray sources obscured in the radio band by matter enshrouding the system.

The optical pulsations observed from PSR J1023+0038 are still open to different interpretations. The pulsed optical luminosity rules out disk reprocessing of accretion-powered X-ray emission and is also hard to reconcile with cyclotron emission from matter accreting onto the neutron star polar caps. We argued that the observed optical pulsed emission could be due to synchrotron emission by relativistic electrons in the magnetosphere of a rotation-powered pulsar. We note that the observed optical efficiency of PSR J1023+0038 exceeds by about three decades that of pulsars of comparable spin-down luminosity. That may be due to the shocked magnetised environment that results from the interaction between the rotation-powered pulsar magnetosphere and the accretion disk, a characteristic of PSR J1023+0038 and likewise other transitional millisecond pulsars. Future observations will assess whether the process responsible for optical pulsations from PSR J1023+0038 coexist or alternate with accretion-powered X-ray pulsations. 


\section{Reference list.}

1. Alpar, M. A., Cheng, A. F., Ruderman, M. A. \& Shaham, J. A new class of radio pulsars. Nature 300, 728-730 (1982).

2. Radhakrishnan, V. \& Srinivasan, G. On the origin of the recently discovered ultra-rapid pulsar. Curr. Sci. India 51, 1096-1099 (1982).

3. Wijnands, R. \& van der Klis, M. A millisecond pulsar in an X-ray binary system. Nature 394, 344-346 (1998).

4. Backer, D. C., Kulkarni, S. R., Heiles, C., Davis, M. M. \& Goss, W. M. A millisecond pulsar. Nature 300, 615-618 (1982).

5. Abdo, A. A. et al. A Population of Gamma-Ray Millisecond Pulsars Seen with the Fermi Large Area Telescope. Science 325, 848 (2009).

6. Archibald, A. M. et al. A Radio Pulsar/X-ray Binary Link. Science 324, 1411 (2009). astro-ph/0905.3397.

7. Papitto, A. et al. Swings between rotation and accretion power in a binary millisecond pulsar. Nature 501, 517-520 (2013). astro-ph/1305.3884.

8. Cocke, W. J., Disney, M. J. \& Taylor, D. J. Discovery of Optical Signals from Pulsar NP 0532. Nature 221, 525-527 (1969). 
9. Deller, A. T. et al. A Parallax Distance and Mass Estimate for the Transitional Millisecond Pulsar System J1023+0038. Astrophys. J. Lett. 756, L25 (2012). astro-ph/1207. 5670.

10. Stappers, B. W. et al. A State Change in the Missing Link Binary Pulsar System PSR J1023+0038. Astrophys. J. 790, 39 (2014). astro-ph/1311. 7506.

11. Patruno, A. et al. A New Accretion Disk around the Missing Link Binary System PSR J1023+0038. Astrophys. J. Lett. 781, L3 (2014). astro-ph/1310.7549.

12. Tendulkar, S. P. et al. NuSTAR Observations of the State Transition of Millisecond Pulsar Binary PSR J1023+0038. Astrophys. J. 791, 77 (2014). astro-ph/1406.7009.

13. Archibald, A. M. et al. Accretion-powered Pulsations in an Apparently Quiescent Neutron Star Binary. Astrophys. J. 807, 62 (2015). astro-ph/1412.1306.

14. Bogdanov, S. et al. Coordinated X-Ray, Ultraviolet, Optical, and Radio Observations of the PSR J1023+0038 System in a Low-mass X-Ray Binary State. Astrophys. J. 806, 148 (2015). astro-ph/1412.5145.

15. Papitto, A. \& Torres, D. F. A Propeller Model for the Sub-luminous State of the Transitional Millisecond Pulsar PSR J1023+0038. Astrophys. J. 807, 33 (2015). astro-ph/1504.05029.

16. Deller, A. T. et al. Radio Imaging Observations of PSR J1023+0038 in an LMXB State. Astrophys. J. 809, 13 (2015). astro-ph/1412.5155. 
17. Takata, J. et al. Multi-wavelength Emissions from the Millisecond Pulsar Binary PSR J1023+0038 during an Accretion Active State. Astrophys. J. 785, 131 (2014). astro-ph/1312.0605.

18. Coti Zelati, F. et al. Engulfing a radio pulsar: the case of PSR J1023+0038. Mon. Not. $R$. Astron. Soc. 444, 1783-1792 (2014). astro-ph/1409.0427.

19. Shahbaz, T. et al. The binary millisecond pulsar PSR J1023+0038 during its accretion state - I. Optical variability. Mon. Not. R. Astron. Soc. 453, 3461-3473 (2015). astro-ph/1507.07473.

20. Jaodand, A. et al. Timing Observations of PSR J1023+0038 During a Low-mass X-Ray Binary State. Astrophys. J. 830, 122 (2016). astro-ph/1610.01625.

21. Davidsen, A., Henry, J. P., Middleditch, J. \& Smith, H. E. Identification of the X-Ray Pulsar in Hercules: a New Optical Pulsar. Astrophys. J. Lett. 177, L97 (1972).

22. Papitto, A. et al. X-ray coherent pulsations during a sub-luminous accretion disc state of the transitional millisecond pulsar XSS J12270-4859. Mon. Not. R. Astron. Soc. 449, L26-L30 (2015). astro-ph/1412.4252.

23. Gierliński, M., Done, C. \& Barret, D. Phase-resolved X-ray spectroscopy of the millisecond pulsar SAX J1808.4-3658. Mon. Not. R. Astron. Soc. 331, 141-153 (2002). astro-ph/0111310.

24. Frank, J., King, A. \& Raine, D. J. Accretion Power in Astrophysics: Third Edition (2002). 
25. Romanova, M. M., Ustyugova, G. V., Koldoba, A. V. \& Lovelace, R. V. E. Three-dimensional Simulations of Disk Accretion to an Inclined Dipole. II. Hot Spots and Variability. Astrophys. J. 610, 920932 (2004). astro-ph/ 0404496.

26. Pacini, F. \& Salvati, M. The optical luminosity of very fast pulsars. Astrophys. J. 274, 369-371 (1983).

27. Romani, R. W. Gamma-Ray Pulsars: Radiation Processes in the Outer Magnetosphere. Astrophys. J. 470, 469 (1996).

28. Mignani, R. P. Optical, ultraviolet, and infrared observations of isolated neutron stars. Adv. Space Res. 47, 1281-1293 (2011). astro-ph/0912.2931.

29. Strader, M. J. et al. Search for optical pulsations in PSR J0337+1715. Mon. Not. R. Astron. Soc. 459, 427-430 (2016). astro-ph/1603.05270.

30. Rangelov, B. et al. Hubble Space Telescope Detection of the Millisecond Pulsar J2124-3358 and its Far-ultraviolet Bow Shock Nebula. Astrophys. J. 835, 264 (2017). astro-ph/1701.00002.

31. Mignani, R. P., Pavlov, G. G. \& Kargaltsev, O. A possible optical counterpart to the old nearby pulsar J0108-1431. Astron. Astrophys. 488, 1027-1030 (2008). astro-ph/0805.2586.

32. Papitto, A., Torres, D. F. \& Li, J. A propeller scenario for the gamma-ray emission of lowmass X-ray binaries: the case of XSS J12270-4859. Mon. Not. R. Astron. Soc. 438, 2105-2116 (2014). astro-ph/1312.0456. 
33. Manchester, R. N., Hobbs, G. B., Teoh, A. \& Hobbs, M. The Australia Telescope National Facility Pulsar Catalogue. Astron. J. 129, 1993-2006 (2005). astro-ph/ 0412641. 
Correspondence and request for materials should be addressed to A. P. (e-mail: alessandro.papitto@oaroma.inaf.it).

Acknowledgements F. A., P. C. and F. M. acknowledge the research project Protocol C26A15YCJ4 funded by the Department of Physics of the University of Rome "La Sapienza", for the financial support. A. P. acknowledges funding from the EUs Horizon 2020 Framework Programme for Research and Innovation under the Marie Skłodowska-Curie Individual Fellowship grant agreement 660657-TMSP-H2020-MSCA-IF2014, and wishes to thank D. de Martino, N. Rea and D. F. Torres for very useful discussions. L. B., T. D.S. and A. P. acknowledge fruitful discussion with the international team on "The disk-magnetosphere interaction around transitional millisecond pulsars" at ISSI (International Space Science Institute), Bern. L. B., T. D.S., G.L. I. and L. S. acknowledge financial contribution from the agreement ASI-INAF I/037/12/0. Results obtained and presented in this paper are based on observations made with the Italian Telescopio Nazionale Galileo (TNG) operated on the island of La Palma by the Fundación Galileo Galilei of the INAF (Istituto Nazionale di Astrofisica) at the Spanish Observatorio del Roque de los Muchachos of the Instituto de Astrofisica de Canarias. The authors gratefully acknowledge time Discretionary Observing Time granted by the TNG Director, Dr. E. Molinari and thank Prof. C. Rossi, for her scientific support and contribution.

Author contributions F.A. and A.P. jointly coordinated and contributed with equal proportion to this study. F.M, F.A. and P.C. conceived and designed the optical photometer. F.A., A.G., L.D.F. and L.R. performed the optical observations. A.P and F.A. analysed the data. A.P. and L.S. wrote the paper. A.P., L.S., T.D.S. and L.B. interpreted the results. All authors read, commented on and approved submission of this article.

Competing Interests The authors declare that they have no competing financial interests. 


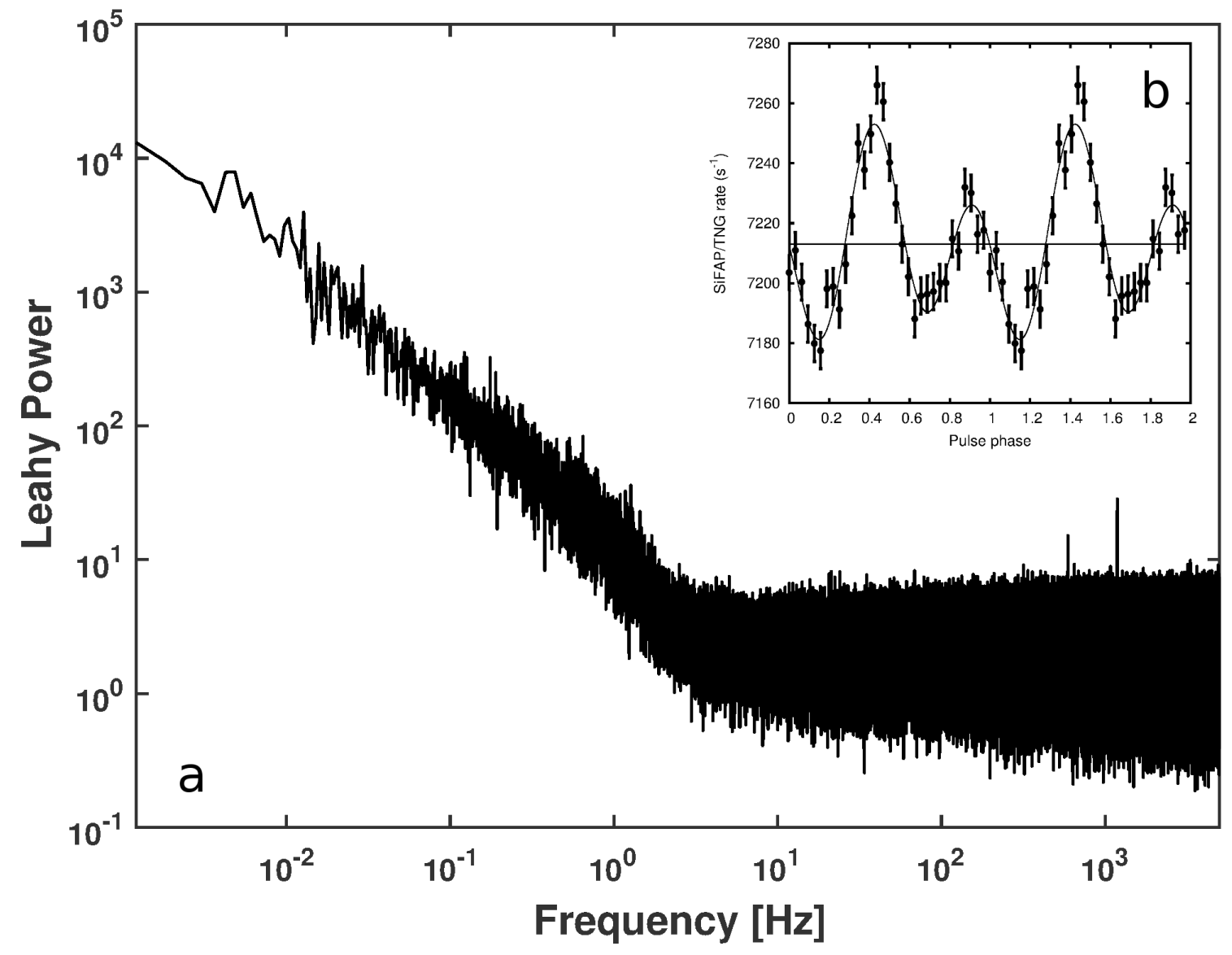

Figure 1: Coherent optical pulsations of PSR J1023+0038. a Average Fourier power spectral density of the 320$900 \mathrm{~nm}$ optical photons observed by SiFAP mounted at the TNG, during four almost consecutive observations starting on March 2, 2016 at 21:40 (UTC), for an exposure of $13.2 \mathrm{ks}$. The power spectrum was obtained sampling the time series at a time resolution of $0.1 \mathrm{~ms}$ and averaging the density measured in eight intervals, each $1.65 \mathrm{ks}-\mathrm{long}$. The time of arrival of optical photons were corrected for a known systematic drift of the SiFAP clock (see Methods), and converted to the barycenter of the Solar System and to the line of nodes of the binary system hosting PSR J1023+0038, using the parameters listed in Table 1 The peaks at 592.4 and $1184.4 \mathrm{~Hz}$ represent the first and the second harmonic of the coherent signal detected at the pulsar spin frequency. b Average, background subtracted pulse profile obtained by folding the optical photons detected during the four TNG observations around $P_{\text {opt }}=1.68798744 \mathrm{~ms}$ (see Table 1 . The pulse profile is sampled by 32 phase bins, error bars show uncertainties at 1- $\sigma$ confidence level, and two cycles are plotted for clarity. The dashed solid line is a Fourier decomposition with two harmonic components with fractional amplitudes $A_{1}=(0.18 \pm 0.02) \%$ and $A_{2}=(0.34 \pm 0.02) \%$ (giving a total amplitude $A=\left(A_{1}^{2}+A_{2}^{2}\right)^{1 / 2}=(0.38 \pm$ $0.03) \%$ ) with respect to the net optical flux of PSR J1023+0038 averaged over an orbital cycle, $K=8185 \pm 76 \mathrm{~s}^{-1}$. The variance of the profile with respect to a constant is $\chi^{2}=438$ for 31 degrees of freedom. The probability of 


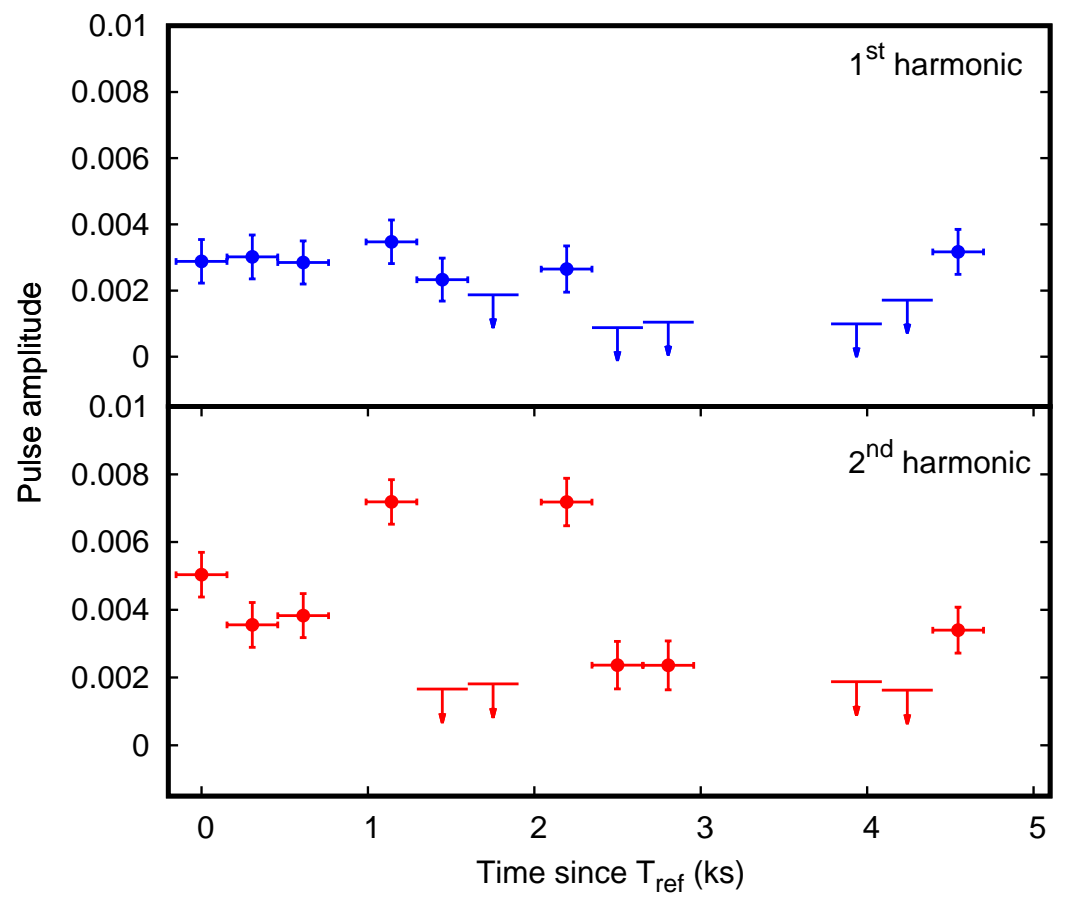

Figure 2: Variability of the coherent optical pulsed amplitude of PSR J1023+0038. Background subtracted fractional amplitudes of the first $\left(A_{1}\right.$; panel a) and second $\left(A_{2}\right.$; panel $\left.\mathbf{b}\right)$ harmonic of pulse profiles obtained folding the SiFAP time series around $P_{\text {opt }}=1.68798744 \mathrm{~ms}$ over $1.1 \mathrm{ks}-$ long intervals, sampling the period in $n=16$ phase bins. The pulse profiles were modelled with the function, $R(t)=K\left\{1+A_{1} \sin \left[2 \pi / P_{\text {opt }}\left(t-\phi_{1}\right)\right]+A_{2} \sin \left[4 \pi / P_{\text {opt }}\left(t-\phi_{2}\right)\right\}\right.$, with $K=8185 \pm 76 \mathrm{~s}^{-1}$, equal to the average net optical flux of PSR J1023+0038, measured by modeling the counting rates observed during the four observations (spanning $4.85 \mathrm{hr}$ ) with a sine function at the orbital period of the binary system, $4.75 \mathrm{hr}$ (see Table 1). Error bars show uncertainties at 1- $\sigma$ confidence level, upper limits were evaluated at the 3- $\sigma$ confidence level. 


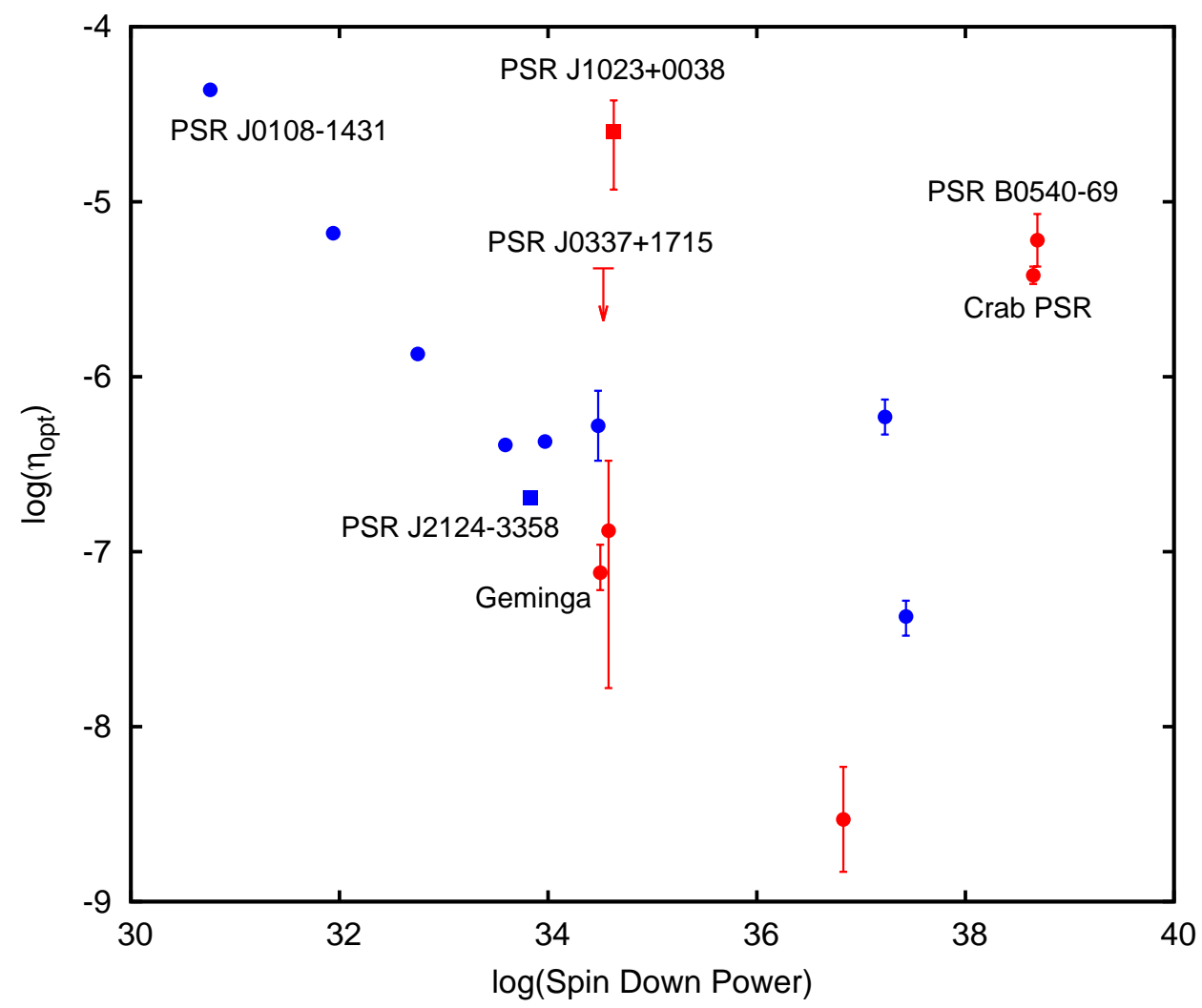

Figure 3: Optical efficiency of rotation-powered pulsars, $\eta_{o p t}=L_{o p t} / \dot{E}_{s d}$ as a function of the pulsar spin down power, $\dot{E}_{s d}$. Red symbols indicate optical pulsars or sources for which an upper limit was set on the pulse amplitude. Blue symbols mark pulsar optical counterparts proposed on the basis of a detection of an (unpulsed) optical source at a position compatible with the pulsar, under the assumption that the optical output traces magnetospheric emission. Circles refer to high-magnetic field $\left(B_{s}>10^{-11} \mathrm{G}\right)$, slow $(P>0.1 \mathrm{~s})$ pulsars, squares are used to mark low-magnetic field $\left(B_{s}<10^{9} \mathrm{G}\right)$ millisecond $(P<0.01 \mathrm{~s})$ pulsars. The optical luminosity was evaluated in the B-band, except for a few cases (see Supplementary Table 1). Error bars show uncertainties at 1- $\sigma$ confidence level. The mean pulsed flux of PSR J1023+0038 was evaluated as $\langle A\rangle=(0.38 \pm 0.03)$ per cent of the average de-reddened B-band magnitude ${ }^{60}$, $16.77 \pm 0.05$. Values of the pulsar distance and spin down power were taken from ATNF pulsar catalog 59 . 
Table 1: X-ray ${ }^{20}$ and optical (this work) ephemerides of PSR J1023+0038.

\begin{tabular}{|c|c|c|}
\hline Parameter & X-rays $s^{20}$ & Optical (this work) \\
\hline Right Ascension(a), $\alpha$ [J2000] & 10:23:47.687198 & $\ldots$ \\
\hline Declination $^{(\mathrm{a})}, \delta[\mathrm{J} 2000]$ & $+00: 38: 40.84551$ & $\ldots$ \\
\hline Reference Epoch, $T_{\text {ref }}$ (MJD) & 57449.9028346 & $\ldots$ \\
\hline Spin Period ${ }^{(b)}, P_{s}(\mathrm{~ms})$ & $1.68798744420(13)$ & $1.68798744(6)$ \\
\hline Orbital Period, $P_{\text {orb }}(\mathrm{s})$ & 17115.5216592 & $17116.1 \pm 5.5$ \\
\hline Projected semi-major axis, $a \sin i / c$ (It-s) & $0.343356(3)$ & $0.3434(1)$ \\
\hline Epoch of ascending node passage ${ }^{(\mathrm{c})}, T_{a s c}$ (MJD) & $57449.7258(3)$ & $57449.72579(9)$ \\
\hline
\end{tabular}

(a) Astrometric position determined from radio interferometr ${ }^{9}$.

(b) Extrapolation to the reference epoch $T_{\text {ref }}$ of the period of the X-ray pulse ${ }^{20}$ estimated at $T_{J}=56458 \mathrm{MJD}$, $P_{0}=1.68798744494252(13) \mathrm{s}$, taking into account the spin period derivative, $\dot{P}=(8.665+/-0.026) \times 10^{-21}$.

(c) Extrapolation to the reference epoch $T_{r e f}$ of the epoch of passage at the ascending node determined from the formal orbital solution that models the epochs of passage at the ascending node determined from X-ray pulsations ${ }^{20}, T_{\text {asc }}=54905.96943473 \mathrm{MJD}, P_{\text {orb }}=17115.5502801 \mathrm{~s}, \dot{P}_{\text {orb }}=(-1.65 \pm 0.19) \times 10^{-10}$. 


\section{Methods}

The optical data set. We observed PSR J1023+0038 with the Silicon Fast Astronomical Photome$\operatorname{ter}^{\sqrt{3435}}$ (SiFAP) mounted at the Nasmyth B focus of the $3.58 \mathrm{~m}$ Telescopio Nazionale Galiled ${ }^{36}$ (TNG), in La Palma Spain, exploiting the focal plane of the Device Optimized for the LOw RESolution (DOLORES) instrument ${ }^{37}$. SiFAP is a 2-channel ultra fast optical photometer developed at the Department of Physics of the University of Rome "La Sapienza". It comprises two Multi Pixel Photon Counters (MPPCs) modules manufactured by Hamamatsu Photonics, one aimed at measuring photon counting rates from the target and the other at monitoring a reference star in the Field of View (FoV). These MPPCs integrate signals in configurable time windows from $100 \mathrm{~ms}$ down to $1 \mathrm{~ms}$ via a standard USB interface. They also provide an analog output which can tag the Time of Arrival (ToA) of individual photons with a time resolution of $25 \mathrm{~ns}$ and a discriminated output capable of counting photons in time bins of $20 \mu \mathrm{s}$, through two independent custom electronic chains. A Global Positioning System (GPS) unit yields a reference time marker via the Pulse Per Second (PPS) signal with 25 ns resolution at $50 \%$ of the rising edge of the pulse itself.

Four observations of PSR J1023+0038 were performed starting on 2016, March 2 at 21:40 (UT), each lasting $3.3 \mathrm{ks}$ (see Supplementary Table 2). A white filter covering the 320-900 nm band was used in all the observations (see Supplementary Fig. 1). The airmass ranged between 1.13 and 1.74, while seeing conditions varied from 0.8 arcsec up to 3 arcsec. The FoV of the sensor is $\sim 50 \operatorname{arcsec}^{2}$ ensuring that signal loss was negligible. A reference star, UCAC4 454$048424(\alpha=10: 23: 48.935, \delta=+00: 44: 52.814, B=16.309$ mag) was also simultaneously 
observed, at a lower time resolution $(1 \mathrm{~ms})$. We estimated the sum of the sky background and of the dark count-rate by observing a region located 25 arcsec away from the target towards the East direction for $120 \mathrm{~s}$ (between the third and the fourth exposure), which yielded an average countrate of $B=8559 \mathrm{~s}^{-1}$, amounting to roughly $\simeq 50 \%$ of the total photons recorded when pointing at the direction of PSR J1023+0038. The contribution of the dark count rate alone was $\sim 2.5 \times 10^{3} \mathrm{~s}^{-1}$.

Temporal Analysis. We considered data taken by the SiFAP at the maximum possible time resolution of $25 \mathrm{~ns}$. A systematic effect introduced a significant difference between the actual photon arrival times and those measured by the SiFAP system quartz clock. In each of the four observations, the total time elapsed between the two GPS-PPS signals marking the beginning and the end of each exposure, which we took as a reference, $\Delta t_{\text {obs }}^{G P S}=3300 \mathrm{~s}$, was slightly longer than the time interval measured by the SiFAP clock, $\Delta t_{\text {obs }}^{S i F A P}=\Delta t_{\text {obs }}^{G P S}+\delta t$, with $\delta t \simeq$ $6 \times 10^{-3} \mathrm{~s}$. We assumed that this difference was due to a constant drift of the time recorded by the SiFAP clock with respect to the actual time, yielding a cumulative linear effect on the recorded times of arrival, $t_{S i F A P}$. We corrected the arrival times by using the following relation, $t_{c o r r}=t_{S i F A P} \times\left(\Delta t_{o b s}^{G P S} / \Delta t_{o b s}^{S i F A P}\right)$. We checked this procedure by applying it to the arrival times recorded during an observation of the Crab pulsar performed with the $1.52 \mathrm{~m}$ Cassini telescope (http://davide2.bo.astro.it/loiano/152 cm-telescope/) of the Bologna Astronomical Observatory on 2016, December 2 at 00:56 (UT), with the same equipment 35 . Using the uncorrected arrival times, a spin period of $P_{C r a b}^{S i F A P}=33.7296127(23)$ ms was obtained; by applying the correction above we recovered a period of $P_{C r a b}^{c o r r}=33.7295827(23) \mathrm{ms}$, fully compatible with that extrapolated from the monthly radio ephemeris provided by the Jodrell Bank Observatory 
(http://www.jb.man.ac.uk/pulsar/crab/crab2.txt), $P_{\text {Crab }}^{J B O}=33.7295810(11) \mathrm{ms}$. This proved the robustness of the SiFAP clock correction. We also determined through laboratory tests (using a frequency/period counter) the maximum jitter of the system quartz clock period $\left(P^{c l k}=8.000009873(1) \times 10^{-9} \mathrm{~s}\right)$ determined by a thermal drift, $\Delta P_{\max }^{c l k}=P_{\max }^{c l k}-P_{\min }^{c l k}=$ $4.8 \times 10^{-15} \mathrm{~s}$. The relative uncertainty on the measured period introduced by the thermal drift is thus $6 \times 10^{-7}$, i.e. about sixty times smaller than the relative accuracy of our determination of the spin period of PSR J1023+0038 (see Table1). The effect of thermal drift could be safely neglected.

We reported the times of arrival of the corrected photons to the Solar System Barycentre, using the JPL DE431 ephemeris (https://ssd.jpl.nasa.gov/horizons.cgi) and the source radio astrometric position ${ }^{16}$. A search for periodicities at the known spin period of the pulsar (see Table 1) was then conducted via the epoch folding search technique 38 . No signal was detected by performing an epoch folding search with $n=16$ phase bins on the four time series not corrected for the pulsar orbital motion, down to an upper limit on the amplitude of $A<10^{-6}$ (3- $\sigma$ confidence level). On the other hand, correcting the times of arrivals of optical photons for the Römer delay caused by the pulsar orbital motion with the ephemerides derived from the X-ray pulsations ${ }^{20}$, allowed us to detect significantly a coherent signal at the pulsar spin period over time intervals as short as $1.1 \mathrm{ks}$. The $\chi^{2}$ of an epoch folding search over a range of periods around the known spin period of the pulsar is plotted in Supplementary Fig. 2. The background subtracted pulse profile was modeled with two Fourier components of fractional amplitude $A_{1}$ and $A_{2}$, correponding to the fundamental and first overtone of the signal (see the inset of Fig. 1 of the main body), $R(t)=K\left\{1+A_{1} \sin \left[2 \pi / P_{\text {spin }}\left(t-\phi_{1}\right)\right]+A_{2} \sin \left[4 \pi / P_{\text {spin }}\left(t-\phi_{2}\right)\right\}\right.$. The mean 
optical flux of PSR J1023+0038 over an orbital cycle, $K=8185 \pm 76 \mathrm{~s}^{-1}$, was determined by modelling with a sine function at the orbital period of the binary system the count-rate observed during the four observations, and binned in time intervals of $50 \mathrm{~s}$. The fractional amplitudes of these two components varied over the $1.1 \mathrm{ks}$ intervals considered, from $A_{1}^{\max }=(0.35 \pm 0.07) \%$ and $A_{2}^{\max }=(0.72 \pm 0.07) \%$, to the non-detection (see Fig. 2). The total pulse amplitude was evaluated as $A=\left(A_{1}^{2}+A_{2}^{2}\right)^{1 / 2}$, and varied around an average value of $\langle A\rangle=0.38 \%$, with a maximum of $A^{\max }=(0.80 \pm 0.07) \%$. The most stringent upper limit on the total pulse amplitude of $A<0.19 \%$ was obtained by folding the first $2.2 \mathrm{ks}$ of the fourth observation.

In order to determine the orbital parameters of the source of the optical photons, we modeled the temporal evolution of the phase of the first harmonic component in terms of the difference between the orbital and spin parameters used to correct the photon arrival times, and the actual ones $^{40}$. The procedure was iterated until no significant corrections to the parameters were found, to within the uncertainties 41 . The spin and orbital parameters we obtained are compatible with those expected from the extrapolation of the ephemeris measured from the X-ray pulsations to the epoch of optical observations (see Tab. 1). The uncertainties on the projected semi-major axis and on the epoch of passage of the source of optical pulses at the ascending node of its orbit allowed us to identify the source of optical photons from within $\delta r \simeq(30-300) / \sin (i) \mathrm{km}$ from the pulsar. The decrease of the signal power as soon as the values of these two parameters are varied from their best-fitting values are shown in Supplementary Fig. 3 and Fig. 4, respectively. This proved that optical pulses came from the same location of the source of X-ray pulsations. 
X-ray observations. The field around PSR J1023+0038 was observed twice by Swifi ${ }^{42}$ (see Supplementary Table 2) within a few days from the TNG observations presented here. Before (seq. 112) and after (seq. 113) the TNG observations, the source was detected by the X-ray Telescope ${ }^{43}$ (XRT) with an unabsorbed 0.3-10 keV X-ray flux of $0.95_{-0.25}^{+0.23} \times 10^{-11}$ and $1.4_{-0.4}^{+0.5} \times 10^{-11} \mathrm{erg} \mathrm{cm}^{-2} \mathrm{~s}^{-1}$, respectively; the X-ray spectrum was described by a power law with photon index $\alpha=1.7_{-0.2}^{+0.5}$ and $\alpha=1.6_{-0.4}^{+0.7}$. The UltraViolet and Optical Telescope ${ }^{44}$ (UVOT) on-board Swift observed the field with the UV filter M2 (centered at $\lambda=224.6 \mathrm{~nm}$ ). A counterpart of PSR J1023+0038 was detected at Vega magnitudes of 16.32(7) and 16.37(8), respectively. Both the observed X-ray and UV fluxes are typical of the accretion disk state of PSR J1023+0038 11 , allowing us to safely conclude that the source was in such a state during the TNG observations performed less than a day before the second Swift observation considered here (id. 113).

Data availability. The barycentered SiFAP data that support the findings of this study are available in the repository figshare with the identifier doi:10.6084/m9.figshare.5341192. 


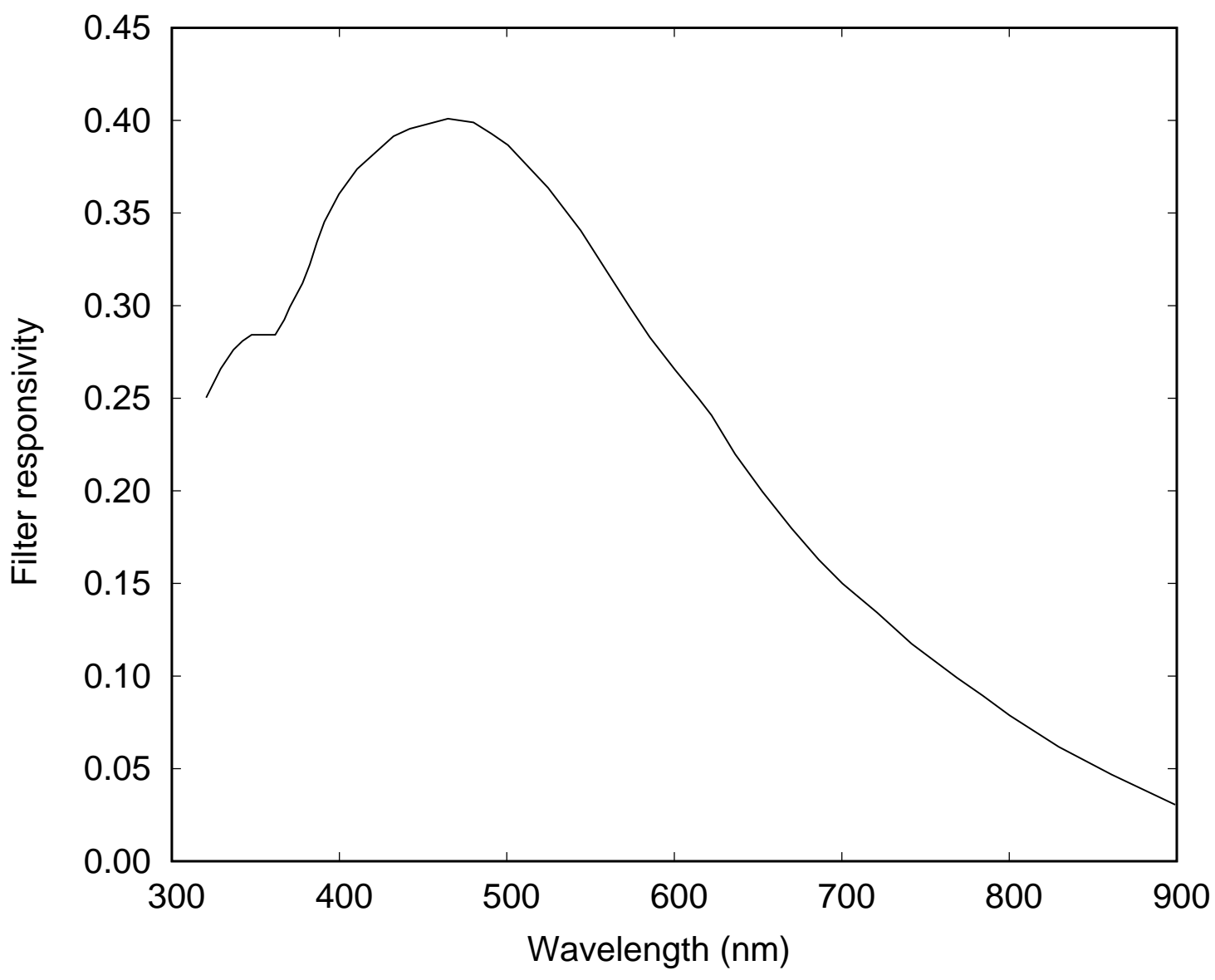

Supplementary Figure 4: Response of the white filter used for all the observations as a function of the wavelength. The distribution reaches a peak value of $\sim 40 \%$ at a wavelength of $450 \mathrm{~nm}$. 


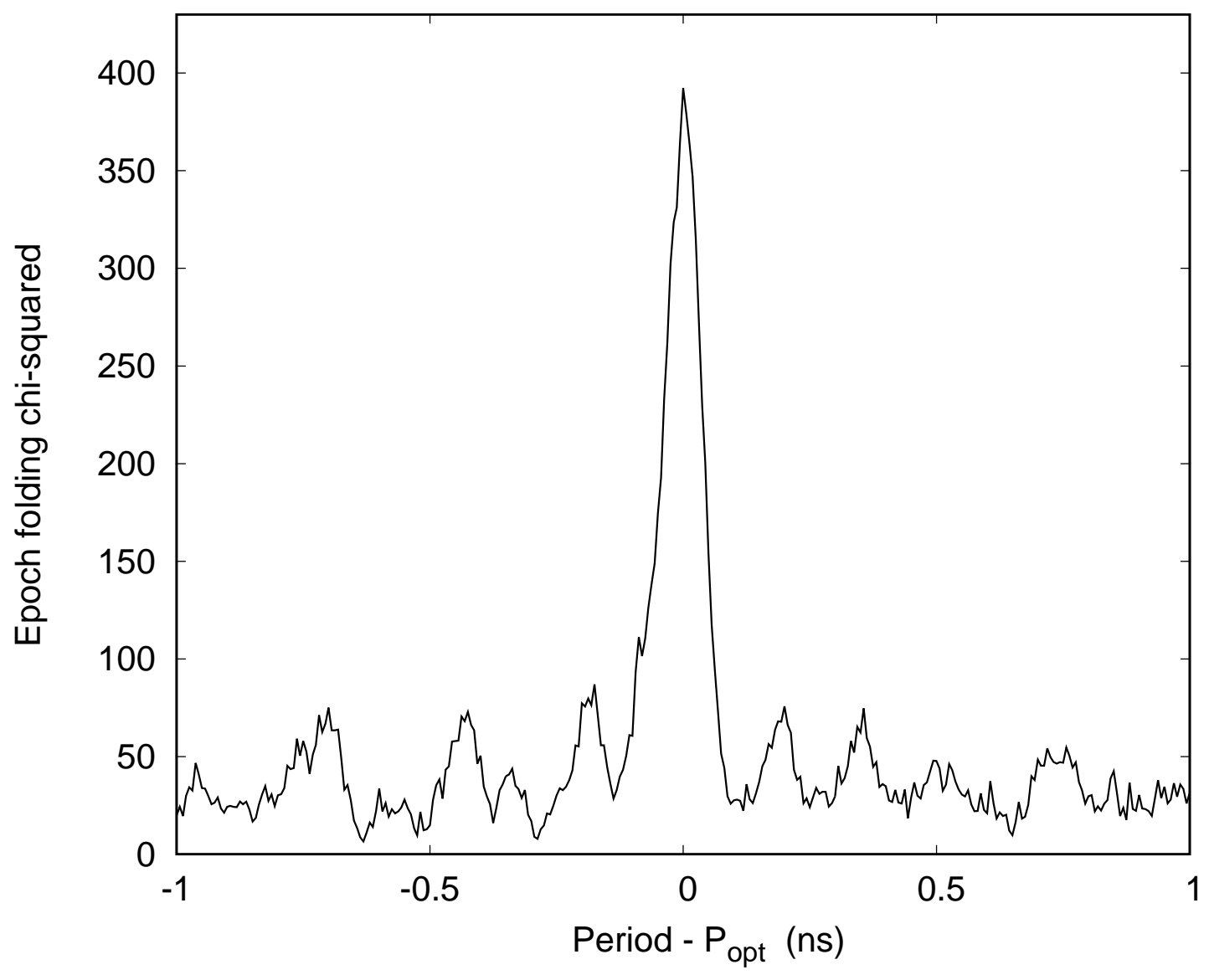

Supplementary Figure 5: Chi-squared versus spin period the epoch folding search performed over the four TNG observation, centered at the best optical period, $P_{o p t}=1.68798744 \mathrm{~ms}$ (see Tab. 1 of the main manuscript), and obtained using $n=16$ phase bins and a period resolution of $5 \times 10^{-12} \mathrm{~s}$. 


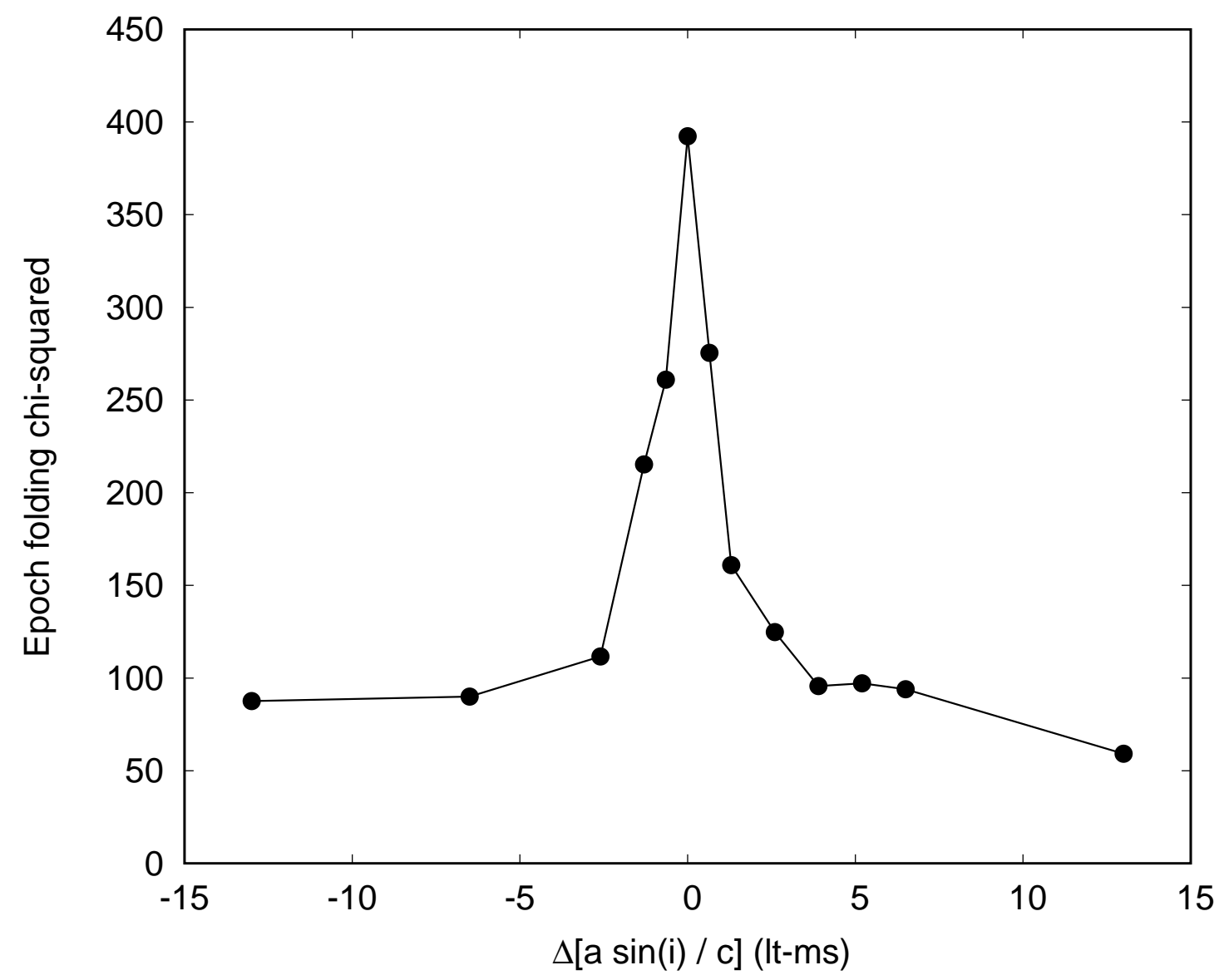

Supplementary Figure 6: Chi-squared of the signal obtained from an epoch folding search around $P_{\text {opt }}$ of the four TNG observations corrected with values of the projected semi-major axis differing by $\Delta[a \sin (i) / c]$ from the best-fitting value (see Tab. 1 of the main manuscript). 


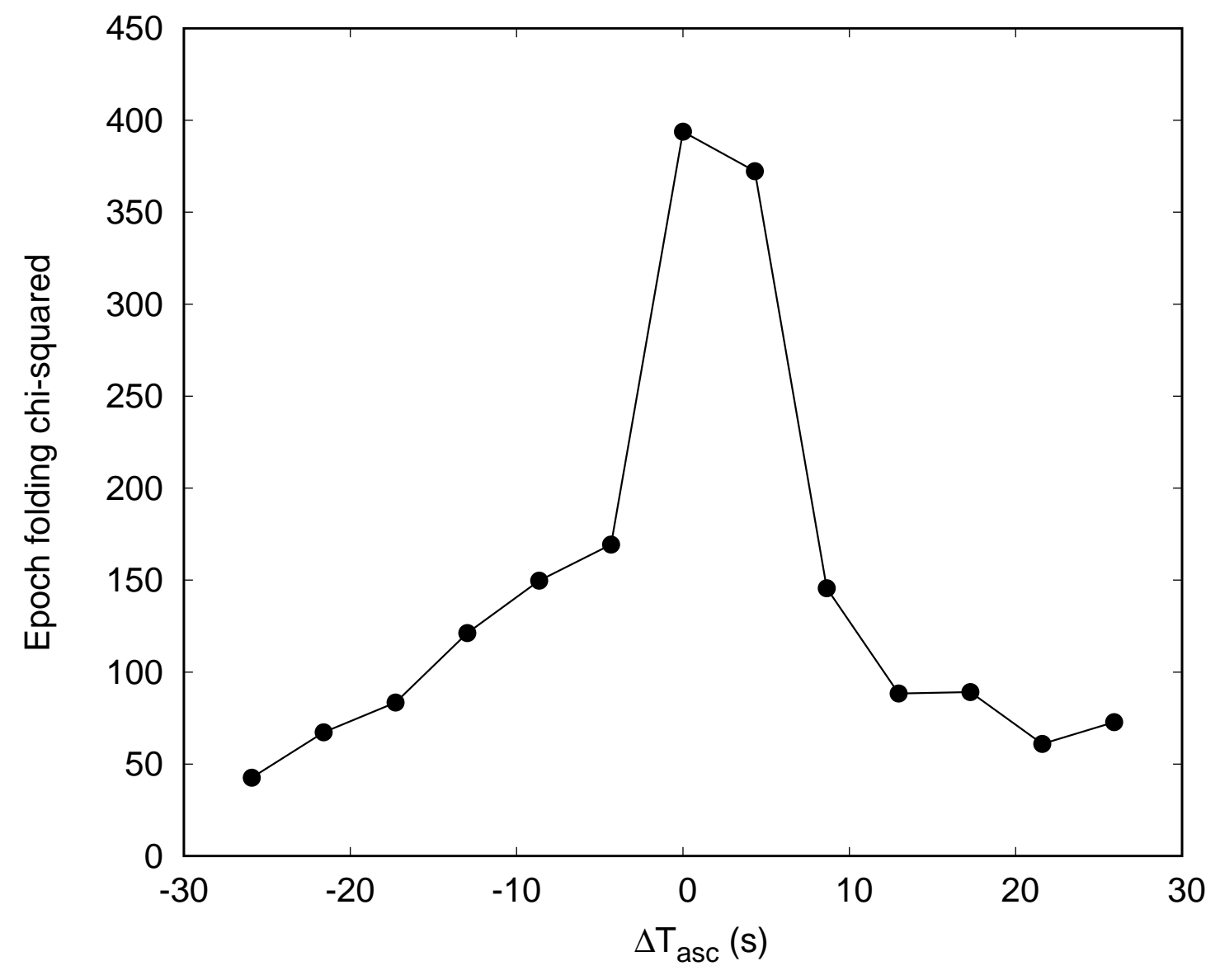

Supplementary Figure 7: Chi-squared of the signal obtained from an epoch folding search around $P_{\text {opt }}$ of the four TNG observations corrected with values of the projected semi-major axis differing by $\Delta\left[T_{\text {asc }}\right]$ from the best-fitting value (see Tab. 1 of the main manuscript). 
Supplementary Table 2: Optical luminosity of rotation-powered pulsars.

\begin{tabular}{|c|c|c|c|c|c|c|}
\hline Pulsar name & $P_{\text {spin }}{ }^{[a]}$ & $\log \tau^{[a]}$ & $\mathbf{d}^{[a]}$ & $\log \dot{E}^{[a]}$ & $\mathbf{B}^{[b]}$ & $\log L_{o p t}$ \\
\hline PSR & s & $\mathrm{yr}$ & $\mathrm{kpc}$ & $\operatorname{erg~s}^{-1}$ & mag & $\operatorname{erg~s}^{-1}$ \\
\hline \multicolumn{7}{|c|}{ Optical Pulsars } \\
\hline B0531+21 (Crab) & 0.033 & 3.1 & 2.0 & 38.65 & $15.27(1)^{45}$ & $33.23(5)$ \\
\hline B0540-69 & 0.051 & 3.2 & 49.7 & 38.69 & $2 2 . 0 ( 2 ) \longdiv { 4 6 }$ & $33.47(15)$ \\
\hline B0833-45 (Vela) & 0.089 & 4.1 & 0.28 & 36.83 & $23.7(3) 47$ & $28.3(3)$ \\
\hline B0656+14 & 0.385 & 5.0 & 0.29 & 34.58 & $25.15(13) 48$ & $27.7_{-0.9}^{+0.4}$ \\
\hline B0633+17 (Geminga) & 0.237 & 5.53 & 0.25 & 34.50 & $25.7(3) 49$ & $27.37_{-0.10}^{+0.16}$ \\
\hline J1023+0038 & $1.69 \times 10^{-3}$ & 9.7 & 1.37 & 34.63 & $22.8_{-0.8}^{+0.5}[c]$ & $30.03_{-0.18}^{+0.33}$ \\
\hline J0337+1715 & $2.73 \times 10^{-3}$ & 9.4 & 1.3 & 34.53 & $>24.5[50 g$ & $<29.15$ \\
\hline \multicolumn{7}{|c|}{ Non-pulsed optical counterparts of pulsars } \\
\hline $\mathrm{J} 2124-3358$ & $4.9 \times 10^{-3}$ & 9.6 & 0.41 & 33.83 & $27.53_{-0.06}^{+0.07} 51 g^{\prime}$ & $27.14(3)$ \\
\hline $\mathrm{B} 1929+10$ & 0.226 & 6.5 & 0.31 & 33.59 & $\geq 26.2 \underline{52}$ & $27.26_{-0.3}^{+0.253}$ \\
\hline B0950+08 & 0.253 & 7.2 & 0.26 & 32.75 & $27.07(16) \underline{53}$ & $26.88(8)$ \\
\hline J0108-1431 & 0.807 & 8.2 & 0.21 & 30.76 & $2 7 . 9 ( 1 ) \longdiv { 5 4 }$ & $26.4(2)$ \\
\hline $\mathrm{B} 1133+16$ & 1.188 & 6.7 & 0.35 & 31.94 & $28.1(3) \square$ & $26.76(17)$ \\
\hline J1741-2054 & 0.414 & 5.6 & 0.30 & 33.98 & $26.45(10)$ & $27.60(5)$ \\
\hline J0205+6449 & 0.066 & 3.7 & 3.2 & 37.43 & $27.4(1) \sqrt[56]{g^{\prime}}$ & $30.06(10)$ \\
\hline B1509-58 & 0.151 & 3.2 & 4.4 & 37.23 & $2 5 . 7 ( 1 ) \longdiv { 5 6 - 5 7 R }$ & $31.0(1)$ \\
\hline B1055-52 & 0.197 & 5.7 & 0.1 & 34.48 & $25.4(1)^{58} \mathrm{~V}$ & $28.2(2)$ \\
\hline
\end{tabular}

${ }^{a}$ Values taken from ATNF pulsar catalog 59

${ }^{b}$ magnitudes are de-reddened and refer to the B band $\left(\lambda_{\text {eff }}=445 \mathrm{~nm}, \Delta \lambda=66 \mathrm{~nm}\right)$, unless otherwise indicated by the superscript;

${ }^{c}$ The equivalent pulsed magnitude of PSR $\mathrm{J} 1023+0038$ in the B-band was evaluated as $\langle A\rangle=3.8 \times 10^{-3}$ times the deredenned B magnitude of the source averaged over an orbital cycle, 16.77(5) mag 60 . The uncertainty reflects the standard deviation of $\sigma_{A}=2.1 \times 10^{-3}$ of the distribution of the amplitudes observed over $1.1 \mathrm{ks}$ intervals (see Methods and Fig. 2 of the the main manuscript). 
Supplementary Table 3: Log of the observations of PSR J1023+0038.

\begin{tabular}{lccc}
\multicolumn{5}{c}{ Telescopio Nazionale Galileo (TNG) } \\
\hline Seq. & Start Time (MJD) & Exposure (s) & Average total countrate $\left(s^{-1}\right)$ \\
1 & 57449.9028371 & 3300 & 15732 \\
2 & 57449.9504008 & 3300 & 15231 \\
3 & 574449.9942661 & 3300 & 18180 \\
4 & 57450.0668420 & 3300 & 16837 \\
\hline \multicolumn{5}{c}{ Swift XRT (Id. 33012) } \\
\hline 112 & 57446.1256713 & 330.9 & 0.27 \\
113 & 57450.9222106 & 200.6 & 0.34 \\
\hline
\end{tabular}




\section{Methods reference list.}

34. Meddi, F. et al. A New Fast Silicon Photomultiplier Photometer. Publ. Astron. Soc. Pac. 124, 448 (2012). astro-ph/1204.3217.

35. Ambrosino, F. et al. The Latest Version of SiFAP: Beyond Microsecond Time Scale Photometry of Variable Objects. J. Astron. Instrum. 5, 16500051267 (2016).

36. Barbieri, C. et al. Status of the Galileo National Telescope. In Stepp, L. M. (ed.) P. Soc. PhotoOpt. Ins., 2199, 10-21 (1994).

37. Zappacosta, L. et al. Constraining the thermal history of the warm-hot intergalactic medium. Astron. and Astrophys. 434, 801-809 (2005). astro-ph/0501402.

38. Leahy, D. A., Elsner, R. F. \& Weisskopf, M. C. On searches for periodic pulsed emission The Rayleigh test compared to epoch folding. Astrophys. J. 272, 256-258 (1983).

39. Leahy, D. A. Searches for pulsed emission - Improved determination of period and amplitude from epoch folding for sinusoidal signals. Astron. Astrophys. 180, 275-277 (1987).

40. Deeter, J. E., Boynton, P. E. \& Pravdo, S. H. Pulse-timing observations of Hercules X-1. Astrophys. J. 247, 1003-1012 (1981).

41. Papitto, A. et al. Spin down during quiescence of the fastest known accretion-powered pulsar. Astron. Astrophys. 528, A55 (2011). astro-ph/1006.1303.

42. Gehrels, N. et al. The Swift Gamma-Ray Burst Mission. Astrophys. J. 611, 1005-1020 (2004). 
43. Burrows, D. N. et al. The Swift X-Ray Telescope. Space Sci. Rev. 120, 165-195 (2005). astro-ph/0508071.

44. Roming, P. W. A. et al. The Swift Ultra-Violet/Optical Telescope. Space Sci. Rev. 120, 95-142 (2005). astro-ph/0507413.

45. Percival, J. W. et al. The Crab pulsar in the visible and ultraviolet with 20 microsecond effective time resolution. Astrophys. J. 407, 276-283 (1993).

46. Middleditch, J., Pennypacker, C. R., Burns, M. S. Optical color, polarimetric, and timing measurements of the 50 MS Large Magellanic Cloud pulsar, PSR 0540-69. Astrophys. J. 315, 142-148 (1987).

47. Nasuti, F. P., Mignani, R., Caraveo, P. A. \& Bignami, G. F. Photometry and proper motion of the VELA pulsar. Astron. Astrophys. 323, 839-843 (1997).

48. Koptsevich, A. B. et al. Optical photometry of the PSR B0656+14 and its neighborhood. Astron. Astrophys. 370, 1004-1016 (2001).

49. Bignami, G. F., Caraveo, P. A. \& Mereghetti, S. The proper motion of Geminga's optical counterpart. Nat. 361, 704-706 (1993).

50. Strader, M. J. et al. Search for optical pulsations in PSR J0337+1715. Mon. Not. R. Astron. Soc. 459, 427-430 (2016).

51. Rangelov, B. et al. Hubble Space Telescope Detection of the Millisecond Pulsar J2124-3358 and its Far-ultraviolet Bow Shock Nebula. Astrophys. J. 835, 264 (2017). 
52. Pavlov, G. G., Stringfellow, G. S. \& Cordova, F. A. Hubble Space Telescope Observations of Isolated Pulsars. Astrophys. J. 467, 370 (1996)

53. Zharikov, S. V. et al. Subaru optical observations of the old pulsar PSR B0950+08. Astron. Astrophys. 394, 633-639 (2002).

54. Mignani, R. P., Pavlov, G. G. \& Kargaltsev, O. A possible optical counterpart to the old nearby pulsar J0108-1431. Astron. Astrophys. 488, 1027-1030 (2008).

55. Zharikov, S. \& Mignani, R. P. On the PSR B1133+16 optical counterpart. Mon. Not. R. Astron. Soc. 435, 2227-2233 (2013).

56. Moran, P. et al. Optical observations of PSR J0205+6449 - the next optical pulsar? Mon. Not. R. Astron. Soc. 436, 401-412 (2013).

57. Wagner, S. J. \& Seifert, W. Optical Polarization Measurements of Pulsars. In IAU Colloq. 177: Pulsar Astronomy - 2000 and Beyond, PASP 202, 315 (2000).

58. Mignani, R. P., Pavlov, G. G. \& Kargaltsev, O. Optical-Ultraviolet Spectrum and Proper Motion of the Middle-aged Pulsar B1055-52. Astrophys. J. 720, 1635-1643 (2010).

59. Manchester, R. N., Hobbs, G. B., Teoh, A. \& Hobbs, M. The Australia Telescope National Facility Pulsar Catalogue. Astron. J. 129, 1993-2006 (2005).

60. Bogdanov, S. et al. Coordinated X-Ray, Ultraviolet, Optical, and Radio Observations of the PSR J1023+0038 System in a Low-mass X-Ray Binary State. Astrophys. J. 806, 148 (2015). 